University of Nebraska - Lincoln

DigitalCommons@University of Nebraska - Lincoln

$11-2007$

\title{
RACE: A Robust Adaptive Caching Strategy for Buffer Cache
}

Yifeng Zhu

University of Maine, zhu@eece.maine.edu

Hong Jiang

University of Nebraska-Lincoln, jiang@cse.unl.edu

Follow this and additional works at: https://digitalcommons.unl.edu/csearticles

Part of the Computer Sciences Commons

Zhu, Yifeng and Jiang, Hong, "RACE: A Robust Adaptive Caching Strategy for Buffer Cache" (2007). CSE Journal Articles. 54.

https://digitalcommons.unl.edu/csearticles/54

This Article is brought to you for free and open access by the Computer Science and Engineering, Department of at DigitalCommons@University of Nebraska - Lincoln. It has been accepted for inclusion in CSE Journal Articles by an authorized administrator of DigitalCommons@University of Nebraska - Lincoln. 


\title{
RACE: A Robust Adaptive Caching Strategy for Buffer Cache
}

\author{
Yifeng Zhu, Member, IEEE, and Hong Jiang, Member, IEEE
}

\begin{abstract}
While many block replacement algorithms for buffer caches have been proposed to address the well-known drawbacks of the LRU algorithm, they are not robust and cannot maintain an consistent performance improvement over all workloads. This paper proposes a novel and simple replacement scheme, called RACE (Robust Adaptive buffer Cache management schemE), which differentiates the locality of $\mathrm{I} / \mathrm{O}$ streams by actively detecting access patterns inherently exhibited in two correlated spaces: the discrete block space of program contexts from which $\mathrm{I} / \mathrm{O}$ requests are issued and the continuous block space within files to which $I / O$ requests are addressed. This scheme combines global $\mathrm{I} / \mathrm{O}$ regularities of an application and local $\mathrm{I} / \mathrm{O}$ regularities of individual files accessed in that application to accurately estimate the locality strength, which is crucial in deciding which blocks are to be replaced upon a cache miss. Through comprehensive simulations on eight realapplication traces, RACE is shown to high hit ratios than LRU and all other state-of-the-art cache management schemes studied in this paper.
\end{abstract}

Index Terms-Operating systems, file systems management, memory management, replacement algorithms.

\section{INTRODUCTION}

$\mathbf{T}$ HIS paper presents a novel approach for buffer cache management, called RACE (Robust Adaptive Caching strategy for buffer cachE), that is motivated by the limitations of existing solutions and the need to further improve the buffer cache hit rate, a significant factor affecting the performance of the supported file system, given the relatively very high buffer cache miss penalties. RACE is shown to overall outperform all existing solutions significantly in most cases. In this section, we first describe the limitations of existing solutions, and then present the main motivations for this work, followed by an outline of the major contributions of the paper.

\section{A. The Limitations of LRU and Recent Solutions, and Motivations}

Designing an effective block replacement algorithm is an important issue in improving file system performance. In most real systems, the replacement algorithm is based on the LeastRecently-Used (LRU) scheme [1], [2] or its clock-based approximation [3]: upon a cache miss, the block whose last reference was the earliest among all cached blocks is replaced. LRU has the advantages of simple implementation and constant space and time complexity. While it has been theoretically verified that LRU can absorb the maximum number of I/O references under a

Yifeng Zhu is with University of Maine, Orono, ME 04469. E-mail: zhu@eece.maine.edu.

Hong Jiang is with University of Nebraska, Lincoln, NE 68588. E-mail: jiang@cse.unl.edu.

Manuscript received 22 May, 2006; revised Jan. 11, 2006; accepted Jun. 28, 2007.

For information on obtaining reprints of this article, please send e-mail to: tc@computer.org, and reference IEEECS Log Number TC-0195-0506. spectrum of workloads that can be represented by the independent reference model [4], in reality LRU often suffers severely from two pathological cases.

- Scan pollution. After a long series of sequential accesses to one-time-use-only (cold) blocks, many frequently accessed blocks may be evicted out from the cache immediately, leaving all these cold blocks occupying the buffer cache for an unfavorable amount of time and thus resulting in a waste of the memory resources. A wise replacement strategy should consider the reference frequency of each block and hence can distinguish hot data from cold data.

- Cyclic access to large working set. A large number of applications, especially those in the scientific computation domain, exhibit a looping access pattern. When the total size of repeatedly accessed data is larger than the cache size, LRU always evicts the blocks that will be revisited in the nearest future, resulting in perpetual cache misses. For example, when repeatedly accessing a file that has 100 blocks, a LRU cache with 99 blocks always evicts the block that will be referenced next and leads to a zero hit ratio. A clever strategy would observe this access with long term locality and only generate cache misses for the references to the block that is least accessed.

To address the limitations of the LRU scheme, several novel and effective replacement algorithms [5]-[8] have been proposed to avoid the two pathological cases described above by using advanced knowledge of the unusual I/O requests. Specifically, they exploit the patterns exhibited in I/O workloads, such as sequential scan and periodic loops, and apply specific replacement polices that can best utilize the cache under that reference pattern.

According to the level at which the reference patterns are observed, these algorithms can be divided into three categories: 1) At the application level, DEAR [6] observes the patterns of references issued by a single application, assuming that the I/O patterns of each application are consistent; 2) At the file level, UBM [5], [9] examines the references to the same file, with an assumption that a file is likely to be accessed with the same pattern in the future. 3) At the program context level, PCC [7] and AMP [8] separate the I/O streams into substreams by program context and detect the patterns in each substream, assuming that a single program context tends to access files with the same pattern in the future.

To best exploit the access patterns, the design space centers around investigating an automatic pattern detection technique that should satisfy the following three requirements.

- Accuracy Applications often have certain I/O access patterns. An accurate detection of access patterns serves as the basis for quantitatively identifying the locality of accessed blocks and tuning caching policies accordingly. A misclassification of an access stream may increase the number of disk 
accesses by evicting useful blocks and taking up memory space. In addition, the detection algorithm should be able to detect not only reference patterns presented explicitly in the consecutive address space but also implicit patterns in a non-consecutive way. For example, a stream of references to blocks with a set of random addresses may belong to a sequential pattern category.

- Responsiveness Real applications within various phases of execution may exhibit different reference patterns. The cache replacement algorithm should adapt to different behaviors within one application and thus a good on-line detection algorithm is required to quickly reflect the transition of access patterns. A detection approach based on aggregate statistical measures of program behavior, as used by PCC and $\mathrm{AMP}$, tends to have a large amount of inertia or reluctance and may not responsively detect local patterns, although it can correctly recognize global patterns.

- Stability A detection-based caching system applies different replacement policies for different reference patterns. To achieve this goal, a buffer cache is divided into multiple partitions and blocks from different patterns are stored in their corresponding partitions. An unstable detection scheme swings a block from different patterns and moves it repeatedly among cache partitions accordingly. In an asynchronous environment with multi-threads, moving a block between the links of different partitions relies on locks to ensure the consistency and correctness, which can be quite costly. A stable classification can eliminate the lock contention and reduce the overhead of cache maintenance.

The key in the design of an effective pattern detection scheme is to strike the optimal tradeoff among the above three requirements. A scheme with better stability may sacrifice its classification accuracy and responsiveness and vice versa. As strongly suggested by the results obtained from the extensive simulations conducted in this study, none of the currently existing schemes is able to maintain a good balance among the three requirements:

1) Application-level detection [6] has good stability but suffers in accuracy and responsiveness since many applications access multiple files and exhibit a mixture of access patterns, as shown in [5] and later in this paper.

2) File-level detection [5], [9] has a smaller observation granularity than the application-based approach but has two main drawbacks that limit its classification accuracy. First, a training process needs to be performed for each new file and thus is likely to cause a misclassification for the references targeted at new files. Second, to reduce the running overhead, the access patterns presented in small files are ignored. Nevertheless, this approach tends to have good responsiveness and stability due to the fact that most files tend to have stable access patterns, although large database files may show mixed access patterns.

3) Program-context-level detection [7], [8] trains only for each program context and has a relatively shorter learning period than the file-based one. While it can make correct classification for new files after training, it classifies the accesses to all files touched by a single program context into the same pattern category, and thus limits the detection accuracy. In addition, it has problems of responsiveness and stability. It bases its decision on aggregate statistical information and thus is not sensitive to pattern changes. The stability problem is caused by the fact that in real applications, as explained in Section III, multiple program contexts may access the same set of files but exhibit different patterns if observed from the program-context point of view.

\section{B. Our Contributions}

This paper makes the following three contributions. First, we collect the I/O traces for ten real applications and investigate I/O access patterns in two correlated spaces: the program context space from which I/O operations are issued, and the file space to which I/O requests are addressed. Second, our comprehensive pattern study in real applications reveals pathological behavior related to existing state-of-the-art cache replacement algorithms including a file-level detection method named UBM [5], [9] and two program context level detection methods named PCC [7] and AMP [8]. This observation motivates us to design a novel, robust and adaptive cache replacement scheme that is presented in this paper. Our new scheme, called RACE which has a time complexity of $\mathrm{O}(1)$, can accurately detect access patterns exhibited in both the discrete block space accessed by a program context and the continuous block space within a specific file, which leads to more accurate estimations and more efficient utilizations of the strength of data locality. We show that our design can effectively combine the advantages of both file-based and program context based caching schemes and best satisfy the requirements of accuracy, responsiveness and stability. Third, we conduct extensive trace-driven simulations by using eight different types of real life workloads and show that RACE substantially improves the absolute hit ratio of LRU by as much asas much as $56.9 \%$, with an average of $15.5 \%$. RACE outperforms UBM, PCC and AMP in absolute hit ratios by as much as $22.5 \%, 42.7 \%$ and $39.9 \%$, with an average of $3.3 \%, 6.6 \%$ and $6.9 \%$, respectively. These gains in absolute hit ratios by RACE are likely to have significant performance implications in applications' response times.

\section{Outline of this Paper}

The rest of this paper is organized as follows. Section II briefly reviews relevant studies in buffer cache management. Section III explains our RACE design in detail. Section IV presents the trace-driven evaluation method and Section $\mathrm{V}$ evaluates the performance of RACE and other algorithms and discusses the experimental results. Finally, Section VI concludes this paper.

\section{Related Work on BUfFER CACHE REPlACEMENT STRATEGIES}

A large number of replacement algorithms have been proposed in the last few decades. These algorithms can be classified into three categories: 1) replacement algorithms that incorporate longer reference histories than LRU, 2) replacement algorithms that rely on application hints, and 3) replacement algorithms that actively detects the I/O access patterns. The following subsections describe the theoretically optimal replacement algorithm, followed by representative replacement algorithms in the above three categories. 


\section{A. Off-line Optimal Replacement}

Off-line optimal policy [10], [11] replaces the block whose next reference is farthest in the future. This policy is not realizable in actual computer systems since it requires complete knowledge of future block references. However, it provides a useful upper bound on the achievable hit ratio of all practical cache replacement policies.

\section{B. Deeper-history Based Replacement}

To avoid the two pathological cases in LRU, described in the previous section, many cache replacement strategies are proposed to incorporate the "frequency" information when making a replacement decision. A common characteristic of such strategies is that all of them keep longer history information than LRU. A chronological list of these algorithms by date of publication includes LRU-K [12], 2Q [13], LRFU [14], EELRU [15], MQ [16], LIRS [17], and ARC [18]. A brief overview of each algorithm is given below.

For every block $x$, LRU-K [12] dynamically records the $K^{\text {th }}$ backward distance, which is defined as the number of references during the time period from the last $K^{t h}$ reference to $x$ to the most recent reference to $x$. A block with the maximum $K^{t h}$ backward distance is dropped to make space for missed blocks. LRU-2 is found to best distinguish infrequently accessed (cold) blocks from frequently accessed (hot) blocks. The time complexity of LRU-2 is $O\left(\log _{2} n\right)$, where $n$ is the number of blocks in the buffer.

2Q [13] is proposed to perform similarly to LRU-K but with considerably lower time complexity. It achieves quick removal of cold blocks from the buffer by using a FIFO queue $A 1_{i n}$, an LRU queue $A m$, and a "ghost" LRU queue $A 1_{\text {out }}$ that holds no block contents except block identifiers. A missed block is initially placed in $A 1_{i n}$. When a block is evicted from $A 1_{i n}$, this block's identifier is added to $A 1_{\text {out }}$. If a block in $A 1_{\text {out }}$ or $A 1_{\text {in }}$ is rereferenced, this block is promoted to $A m$.

LRFU (Least Recently/Frequently Used) [14], [19] endeavors to replace a block that is both least recently and least frequently used. A weight $C(x)$ is associated with every block $x$ and a block with the minimum weight is replaced.

$$
C(x)=\left\{\begin{aligned}
1+2^{-\lambda} C(x) & \text { if } x \text { is referenced at time } t \\
2^{-\lambda} C(x) & \text { otherwise. }
\end{aligned}\right.
$$

where $\lambda, 0 \leq \lambda \leq 1$, is a tunable parameter and initially $C(x)=0$. LRFU reduces to LRU when $\lambda=1$, and to LFU when $\lambda=$ 0 . By controlling $\lambda$, LRFU represents a continuous spectrum of replacement strategies that subsume LRU and LFU. The time complexity of this algorithm ranges between $O(1)$ and $O(\log n)$, depending on the value of $\lambda$.

EELRU (Early Eviction LRU) [15] builds a history queue that records the identifiers of recently evicted blocks and uses this queue to detect the recency of evicted blocks. Based on the recency distributions of referenced blocks, it dynamically changes its replacement strategies. Specifically, it performs LRU replacement by default but diverges from LRU and arbitrarily evicts some pages early to allow not-recently-touched blocks to remain longer when EELRU detects that many pages fetched recently have just been evicted.

MQ (Multi-Queue Replacement Algorithm) [16] uses $m+1$ LRU queues (typically $m=8$ ), $Q_{0}, Q_{1}, \ldots, Q_{m-1}$ and $Q_{o u t}$, where $Q_{i}$ contains blocks that have been referenced at least $2^{i}$ times but no more than $2^{i+1}$ times recently, and $Q_{\text {out }}$ contains the identifiers of blocks evicted from $Q_{0}$ in order to remember access frequencies. On a cache hit in $Q_{i}$, the frequency of the accessed block is incremented by 1 and this block is promoted to the most recently used position of the next level of queue if its frequency is equal to or larger than $2^{i+1}$. MQ associates each block with a timer that is set to currentTime +lifeTime. lifeTime is a tunable parameter that is dependent upon the buffer size and workload. It indicates the maximum amount of time a block can be kept in each queue without any access. If the timer of the head block in $Q_{i}$ expires, this block is demoted into $Q_{i-1}$. The time complexity of MQ is $O(1)$.

LIRS (Low Inter-reference Recency Set) [17], [20] uses the distance between the last and second-to-the-last references to estimate the likelihood of the block being re-referenced. It categorizes a block with a large distance as a cold block and a block with a small distance as a hot block. A cold block is chosen to be replaced on a cache miss. LIRS uses two LRU queues with variable sizes to measure the distance and also provides a mechanism to allow a cold block to compete with hot blocks if the access pattern changes and this cold block is frequently accessed recently. The time complexity of LIRS is $O(1)$. Clock-pro [21] is an approximation of LIRS.

For a given cache size $c$, ARC (Adaptive Replacement Cache) [18], [22] uses two LRU lists $L_{1}$ and $L_{2}$, and they combinatorially contain $c$ physical pages and $c$ identifiers of recently evicted pages. While all blocks in $L_{1}$ have been referenced only once recently, those in $L_{2}$ have been accessed at least twice. The cache space is allocated to the $L_{1}$ and $L_{2}$ lists adaptively according to their recent miss ratios. More cache space is allocated to a list if there are more misses in this list. The time complexity of ARC is $O(1)$. CAR [23] is a variant of ARC based on clock algorithms.

All the above replacement algorithms base their cache replacement decisions on a combination of recency and reference frequency information of accessed blocks. However, they are not able to explicitly exploit the regularities exhibited in past behaviors or histories, such as looping or sequential references. Thus their performance is confined due to their limited knowledge of I/O reference regularities [7].

\section{Application-controlled Replacement}

Application-informed caching management schemes are proposed in ACFS [24] and TIP [25], and they rely on programmers to insert useful hints to inform operating systems of future access patterns. ACFS uses a two-level cache scheme, where a global cache management policy decides which application should give up a cache block upon a miss and the local policy decides intelligently which block of that application should be evicted by applying application-specific knowledge. TIP partitions the cache into three logical domains for hinted-prefetching blocks, hinted-caching blocks, and unhinted-caching blocks, respectively. Based on the estimated cost-benefits, TIP dynamically allocates file buffers among those three domains. To free the programmer from the onerous burden, Profet [26] exploits a compiler-based technique to automatically insert the crucial hints to facilitate $\mathrm{I} / \mathrm{O}$ prefetching. However, this technique cannot achieve satisfactory performance level if the $\mathrm{I} / \mathrm{O}$ access pattern is only known at runtime. Artificial intelligence tools [27] are proposed to learn 
these I/O patterns at execution time and thus obtain the hints dynamically.

\section{Active Pattern-detection Based Replacement}

Depending on the level at which patterns are detected, the pattern-detection based replacement can be classified into four categories: 1) block-level patterns, 2) application-level patterns, 3) file-level patterns, and 4) program-context-level patterns. An example of block level pattern detection policy is SEQ [28], which detects the long sequences of page cache misses and applies the Most-Recently-Used(MRU) [29] policy to such sequences to avoid scan pollution.

At the application level, DEAR (Detection Adaptive Replacement) [6] periodically classifies the reference patterns of each individual application into four categories: sequential, looping, temporally-clustered, and probabilistic. DEAR uses MRU as the replacement policy to manage the cache partitions for looping and sequential patterns, LRU for the partition of the temporallyclustered pattern, and LFU for the partition of the probabilistic pattern. The time complexity of DEAR is $O(n \log n)$ where $n$ is the number of distinct blocks referenced in the detection period.

At the file level, the UBM (Unified Buffer Management) [5], [9] scheme separates the I/O references according to their target files and automatically classifies the access pattern of each individual file into one of three categories: sequential references, looping references and other references. It divides the buffer cache into three partitions, one for blocks belonging to each pattern category, and then uses different replacement policies on different partitions. For blocks in the sequentially-referenced partition, MRU replacement policy is used, since those blocks are never revisited. For blocks in the periodically referenced partition, a block with the longest period is first replaced and the MRU block replacement is used among blocks with the same period. For blocks that belong to neither the sequential partition nor the looping partition, a conventional algorithm, such as LRU, is used.

At the program context level, the Program Counter based Cache (PCC) [7] algorithm exploits virtual program counters exhibited in application's binary execution codes to classify the program signatures into the same three categories as UBM and then uses the same replacement policies for these categories respectively. While UMB classifies the I/O access patterns based on files, PCC classifies the patterns based on the virtual program counters of the I/O instructions in the program code. Adaptive MultiPolicy caching scheme (AMP) [8] inherits the design of PCC but proposes a new pattern detection algorithm. It defines an experiential mathematical expression to measure recency and classifies program counters according to the comparison between the average recency and a static threshold.

\section{The Design of A Robust Adaptive Caching RePlaCEMENT (RACE) Algorithm}

This section presents the design of the RACE caching algorithm in detail. We first introduce the recently developed ProgramContext-based technology in buffer caching and then analyze its limitations that in part motivate our RACE design, which is followed by the presentation of the details of our RACE algorithm.

\section{A. PC-based Technology in Caching Replacement}

Temporal locality of I/O references in all kinds of program executions has been extensively demonstrated and is a well known program behavior. Cache performance can be enhanced by taking full advantage of temporal locality. Based on this principle, many cache management algorithms, including PCC [7] and AMP [8], exploit temporal locality by using history information of program behavior to estimate the reuse distance of cache blocks. These studies successfully link the past $\mathrm{I} / \mathrm{O}$ behavior to their future reoccurrences by borrowing a computer architectural concept: program counters, which indicates the location of the instructions in memory. It is found that a particular instruction, identified by its program counter, usually performs a very unique task and seldom changes its behavior. Thus these studies assume that there is a considerably high probability of the access pattern of a program counter remaining unchanged in the near future.

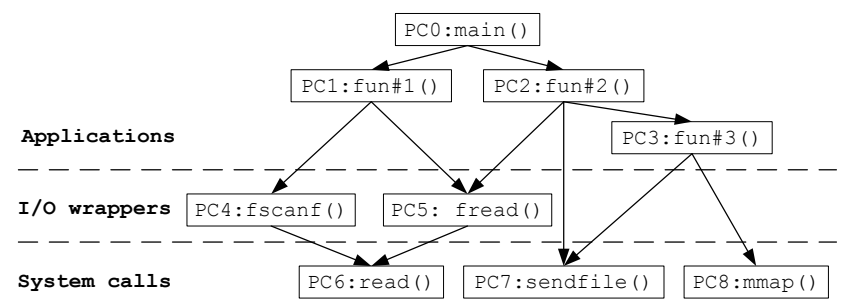

Fig. 1. An example call graph of some application.

Figure 1 presents a call graph to further illustrate the key idea behind the studies of PCC and AMP. A call graph represents the runtime calling relationships among a program's functions or procedures, in which a node corresponds to a function and an arc represents a call. An I/O instruction, issued by some function in the application layer, may be interpreted in the $\mathrm{I} / \mathrm{O}$ wrapper layer that hides the $\mathrm{I} / \mathrm{O}$ complexity and provides flexible interfaces, and eventually invokes system calls to access data. To uniquely identify the program context from which an $\mathrm{I} / \mathrm{O}$ operation is invoked, a program counter signature is defined as the sum of the program counters of all functions along the I/O call path. Program counter signatures can be obtained by traversing the function stack frames backwards from the system calls main(). For simplicity, program signatures are denoted as PCs in the rest of this paper.

PCC and AMP separate the I/O references into sub-streams according to their PCs and then classify PCs into appropriate I/O reference pattern categories. A PC is assumed to exhibit the same I/O reference pattern in the future as it has been classified, and the target data blocks referenced by the current PC will be managed by the corresponding policy. Unfortunately, such an approach has three significant disadvantages.

1) The first iteration of each new PC in a global looping pattern will be misclassified as sequential. This is caused intrinsically by the inability of PC based schemes to detect the phenomenon of pattern sharing among multiple PCs. Pattern sharing in real applications is not rare. For example, it is highly likely that a subroutine is called by multiple parent subroutines but shares the same I/O access pattern. Recursive functions are another example since they generate a set of different program signatures but share the same reference patterns. Multi-threading can also lead to pattern sharing. Figure 2 and 3 show the traces of gnu- 


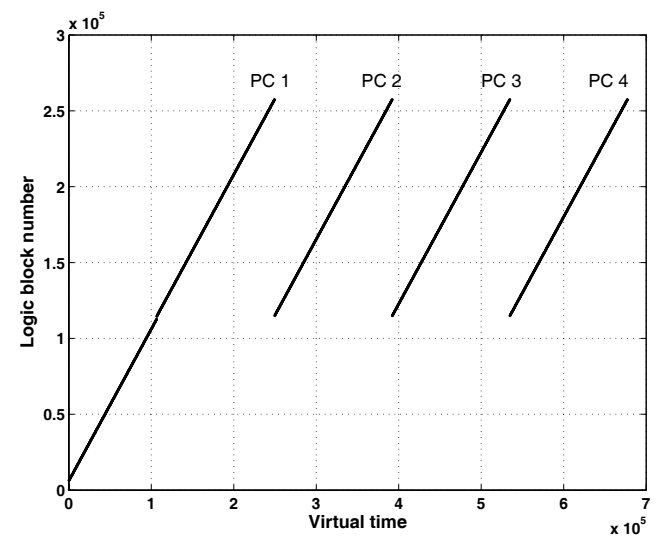

Fig. 2. Block references of gnuplot.

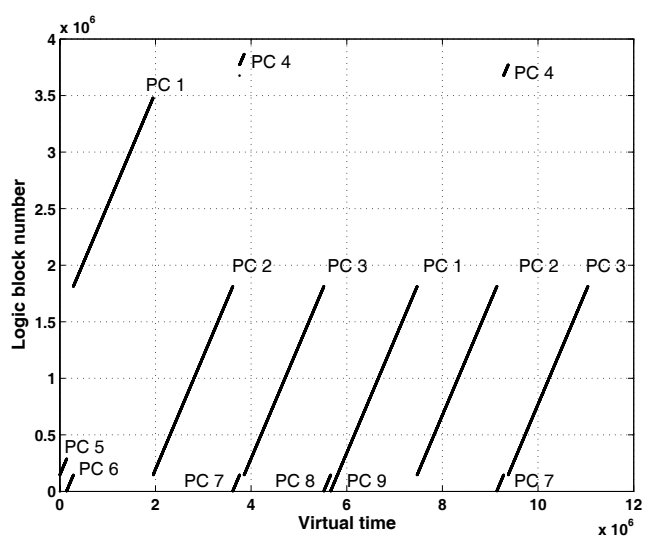

Fig. 3. Block references of BLAST.

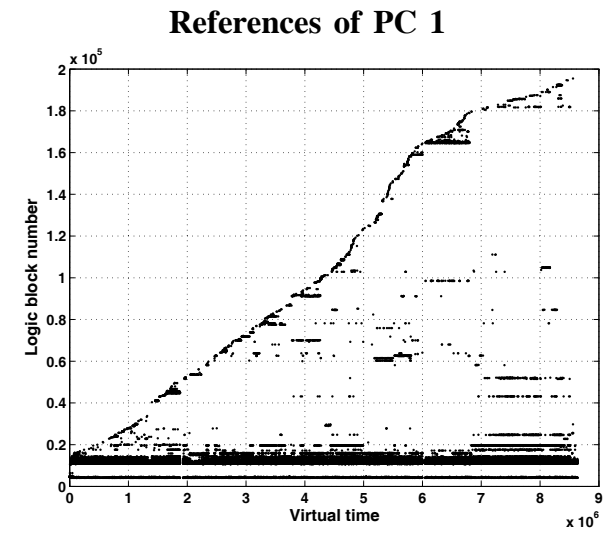

References of PC 4

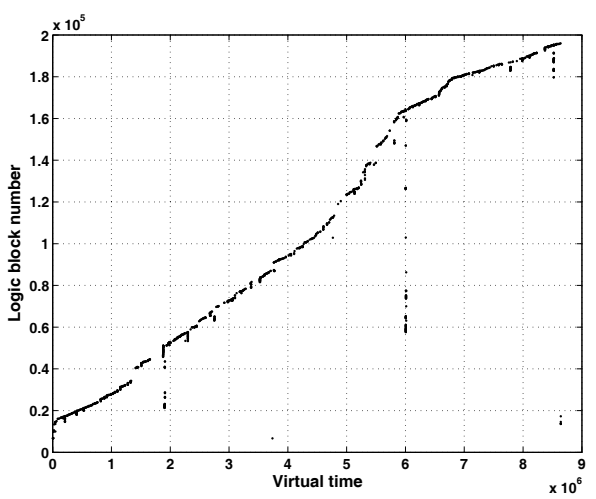

References of PC 2

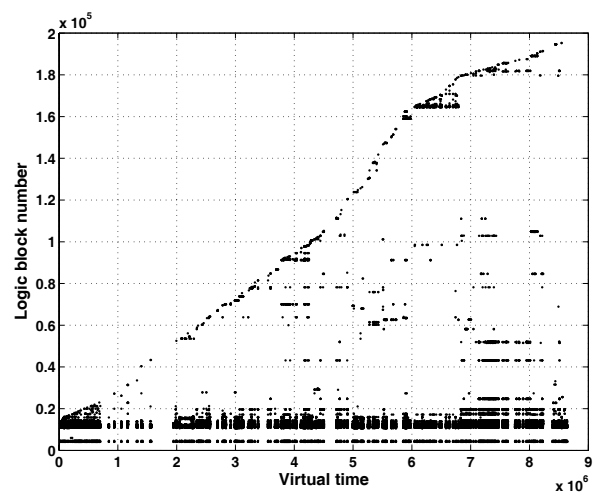

References of PC 5

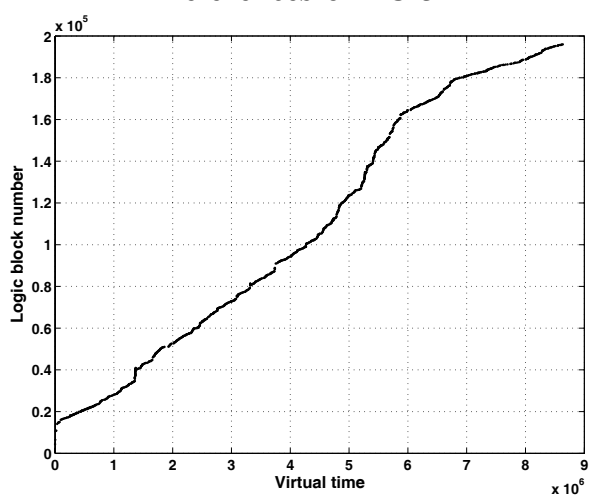

References of PC 3

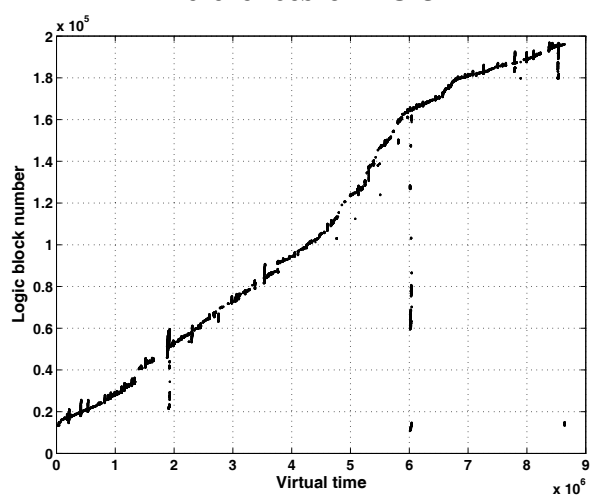

References of PC 6

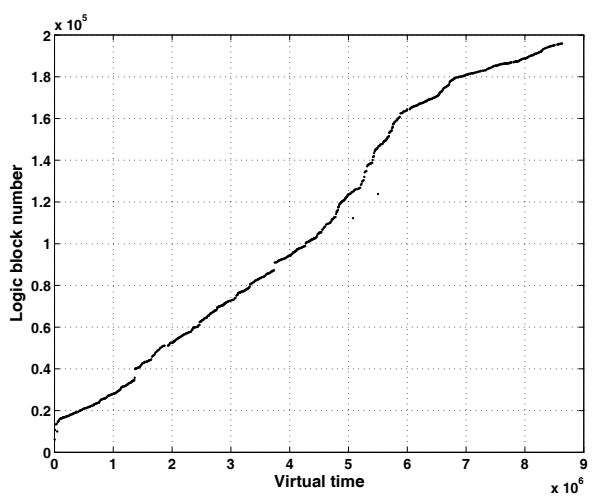

Fig. 4. Traces of 6 PCs with the highest numbers of references in gcc. The PCs, in order of decreasing numbers of references, are from 1 to 6.

plot and BLAST whose detailed descriptions are presented in Section IV. (Throughout this paper, the terms "block number" and "block address" are used interchangeably.) In the gnuplot trace, a sequence of plotting commands are issued in the order of plot, plot, replot, plot $3 d$, replot to two large data files. While the two replot functions access a data file with the same pattern as their previous plot and plot $3 d$ functions, they follow a slightly different I/O path and thus have different PCs with each of the two plots. Although these plotting functions access the data repeatedly, a pure program counter based scheme, such as PCC or AMP, will erroneously classify these references as sequential.
In the trace of BLAST (also described in Section IV), shown in Figure 3, the program forks three threads and searches through the database files simultaneously. While the database files are accessed repeatedly, program-counter based detectors will not be able to classify the patterns correctly due to their inability to retain the "global picture".

2) Pattern conflicts reduce detection accuracy and increase management overhead. Figure 4 presents traces of the top six PCs with the highest numbers of references, collected from the $g c c$ trace described in Section IV. While PC1 and PC2 exhibit a looping pattern, PC5 and PC6 show a sequential pattern and PC3 and PC4 show a sequential 
pattern with a small degree of repetition. Most referenced blocks thus are classified as sequential if they are initiated by PC3, PC4, PC5 and PC6, and as looping if by PC1 and $\mathrm{PC} 2$. Since the references of these PCs are interwoven with one another, blocks need to be continuously moved between the sequential partition and the looping partition. Such moves require locks to ensure consistency and correctness and can cause a significant maintenance overhead.

3) PC-based schemes cannot accurately distinguish locality strengths. Locality strength in the PC-based approach is used to determine which block is replaced. It is measured by the looping period, where a longer looping period represents a weaker locality. PCC uses a single period to measure the locality of all the blocks accessed by a particular PC on a cache miss, and evicts the block accessed by the PC that has the longest looping period. Although this period is averaged exponentially ${ }^{1}$ by weighing recent periods more heavily than older ones, apparently a single looping period will not accurately measure the locality strength when a large amount of data is accessed. This can be easily observed from the traces of PC1 and PC2 in Figure 4. While PC1 and PC2 show a looping pattern, there is a significant portion of blocks whose actual looping periods are much longer than the average looping period.

\section{B. The Design of RACE}

Our RACE scheme is built upon the assumption that future access patterns have a strong correlation with both the program context identified by program signatures and the past access behavior of current requested data. While UBM only associates its prediction with the data's past access behavior, PCC and AMP only consider the relationship between future patterns and the program context in which the current $\mathrm{I} / \mathrm{O}$ operation is generated. Our assumption is more appropriate for real workloads, as demonstrated by our comprehensive experimental study presented in Section V.

Our RACE scheme automatically detects an access pattern as belonging to one of the following three types:

- Sequential references: All blocks are referenced one after another and never revisited again;

- Looping references: All blocks are referenced repeatedly with a regular interval;

- Other references: All references that are not sequential or looping.

Figure 5 presents the overall structure of the RACE caching scheme. RACE uses two important data structures: a file hash table and a PC hash table. The file hash table records the sequences of consecutive block references and is updated for each block reference. The sequence is identified by the file description (inode), the starting and ending block numbers, the last access time of the first block, and their looping period. The virtual access time is defined on the reference sequence, where a reference represents a time unit. The looping period is exponentially averaged over the virtual time. The $P C$ hash table records how many unique blocks each PC has accessed (fresh) and how many references (reused) each PC has issued to access blocks that have been visited previously. Although PCC also uses

\footnotetext{
${ }^{1}$ The exponential average $S$ of a time series $u(t)$ is defined as $S(t+1)=$ $\alpha \cdot S(t)+(1-\alpha) \cdot u(t+1)$ where $0<\alpha<1$.
}

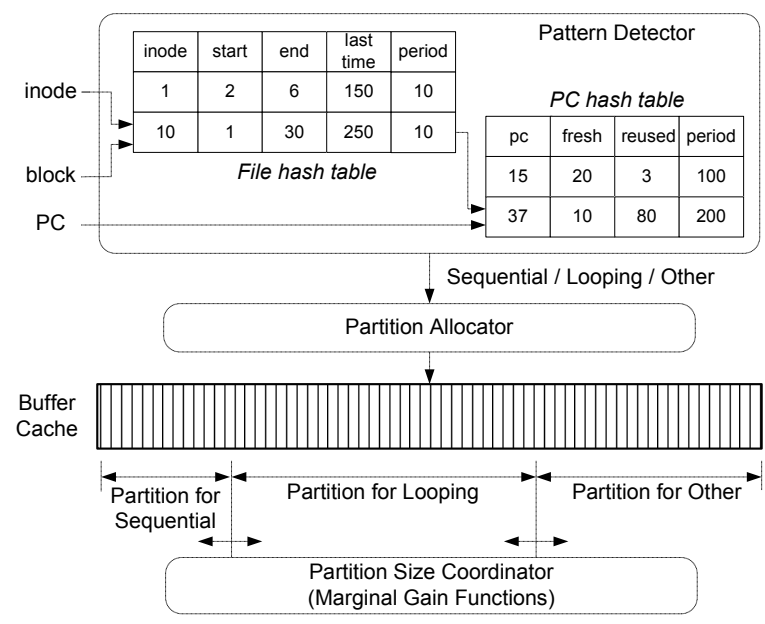

Fig. 5. The key structure of the RACE scheme. The Partition allocator and the Partition size coordinator take the results of pattern detector to adaptively fine-tune the size of each cache partition. If a sequence is found in the file hash table, then the period stored in the file hash table is used to update the period field in the PC hash table.

two counters, our RACE scheme is significantly different from PCC in that: 1) PCC's counters do not accurately reflect the statistical status of each PC process, resulting in misclassification of access patterns, as discussed later in this section, and 2) PCC only considers the correlations between the last $\mathrm{PC}$ and the current PC that accesses the same data block. In fact, many PCs exist in one application and it is likely more than two PCs access the same data blocks.

The detailed pattern detection algorithm is given in Algorithm 1. The main process can be divided into three steps. First, the file hash table is updated for each block reference. RACE checks whether the accessed block is contained in any sequence in the file hash table. If found, RACE updates both the last access time and the sequence's average access period. When a block is not included in any sequence of the file hash table, RACE then tries to extend an existing sequence if the current block address is the next block of that sequence or otherwise RACE assumes that the current request starts a new sequence. Second, RACE updates the PC hash table by changing the fresh and reused counters. For each revisited block, fresh and reused of the corresponding PC are decreased and increased, respectively. On the other hand, for a block that has not been visited recently, the fresh counter is incremented. The last step is to predict access patterns based on the searching results on the file and PC hash tables. If the file table reports that the currently requested block has been visited before, a "looping" pattern is returned. The looping period will identify how often this file have been accessed. If the file table cannot provide any history information of the current block, RACE relies on the PC hash table to make predictions. A PC with its reused counter larger than its fresh counter is considered to show a "looping" pattern. On the other hand, a PC is classified as "sequential" if the PC has referenced a certain amount of one-time-use-only blocks and as "others" if there are no strongly supportive evidence to make a prediction. By using the hashing data structure to index the file and PC tables, which is also used in LRU to facilitate the search of a block in the LRU stack, RACE can be implemented with a time complexity of $O(1)$.

How to efficiently calculate the average access time for each 

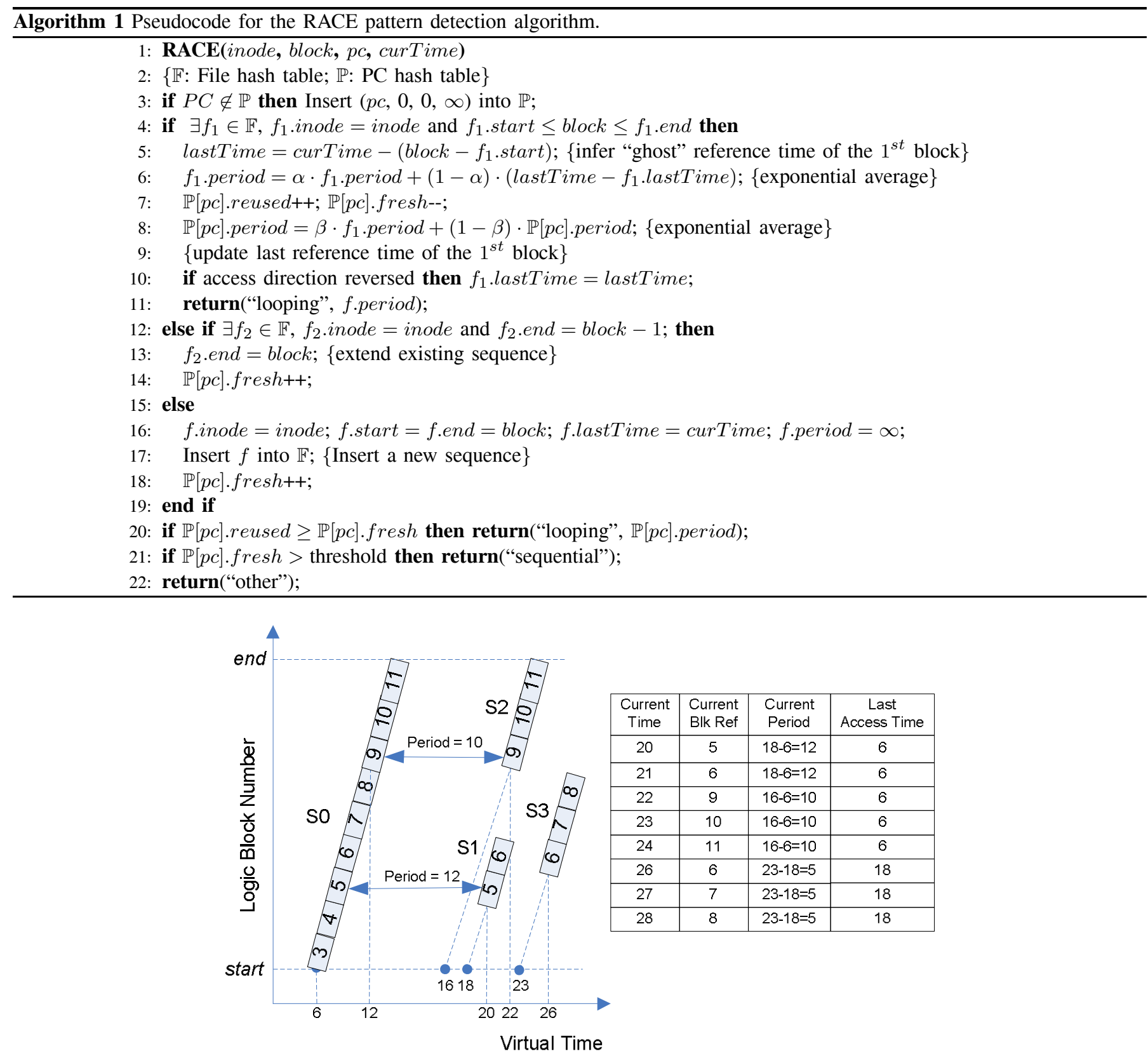

Fig. 6. An example to illustrate the calculation of average access period.

access sequence is a challenging issue. It is not realistic to record the last access time of every accessed block due to prohibitively high overhead. We choose to only record the last access time of the very first block to reduce the overhead. The real implementation is slightly different than the abstract procedure given in Algorithm 1 and Figure 6 shows an example that illustrates the basic process. In a repeated I/O stream, such as $S 1, S 2$ and $S 3$, the access time of each block reference is virtually projected back to the time of the start block. Then the current access period is the time difference between the projected time and the recorded last access time of the start block. The access period of a sequence is exponentially averaged over the access periods of all block references in that sequence. After the second reversing point (a decrement in access addresses), such as the time instant 16 in this example, the last access time recorded in the file hash table is then updated for all subsequent reversing points. Occasionally, the access periods may be erroneously calculated. For example, the access period of references to block 7 and 8 are incorrectly reported as 5. This approach, however, does not compromise the accuracy significantly, as indicated by our simulation results presented in Section V.

The number of repeatedly access blocks, rather than the number of repeatedly accessed files, is used to identify the loop patterns for each PC. This is because we want to make cold files, which are less frequently accessed, weight less in the classifying process. Previous studies have shown that a small fraction of files absorb most of the I/O activities in a file system [30]-[33]. We believe that this commonly existed file access locality justifies our choice. Otherwise, biased pattern predictions would be generated if we place the same weight on all files and do not consider how many 


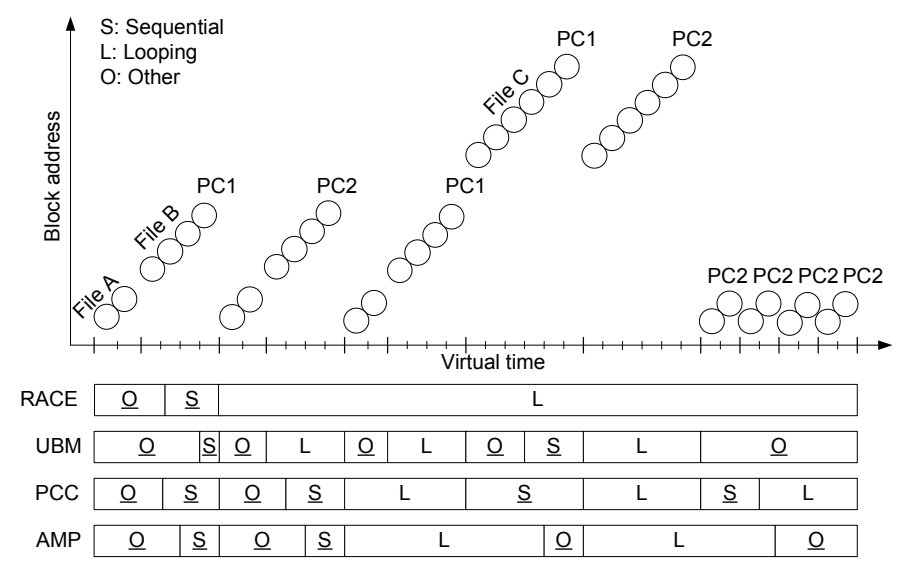

Fig. 7. An example of reference patterns. The sequentiality thresholds for UBM, PCC and RACE are 3. The sequentiality threshold, looping threshold and exponential average parameter for AMP are 0.4, 0.01, and 4 respectively. All incorrect classification results are underscored.

blocks have been accessed from individual files.

By observing the patterns both at the program context level and the file level and by exploiting the detection mechanism in both the continuous block address space within files and the discrete block address space in program contexts, RACE can more accurately detect access patterns. An example, shown in Figure 7, is used to illustrate and compare the classification results of RACE, UBM, PCC, and AMP, in which all false classification results are underscored.

$\boldsymbol{R A C E}$ File A is initially classified as other. After File A is visited, the fresh and reused counters of $\mathrm{PC} 1$ are set to 2 and 0 respectively. After the first block of File B is accessed, the pattern of PC1 immediately changes to be sequential since the fresh count becomes larger than the threshold. Thus during the first iteration of accesses to File A and B, RACE incorrectly classifies the first three blocks as other and then next three blocks as sequential. However, after the first iteration, RACE can correctly identify the access patterns. During the second and third iterations, the sequences for both File A and File B are observed in the file hash table and are correctly classified as looping. Although File $\mathrm{C}$ is visited for the first time, it is still correctly classified as looping. This is because the fresh and reused counters of PC1 are 0 and 6 respectively before File $\mathrm{C}$ is accessed. After that, all references are made by $\mathrm{PC} 2$ and they are classified as looping since the file hash table have access records of File $\mathrm{B}$ and $\mathrm{C}$.

$\boldsymbol{U B M}$ Since the total number of blocks in File A is less than the threshold in UBM, all references to File A are incorrectly classified as other. The initial references to the first three blocks and the fourth block of File B are detected as other and sequential, respectively. After that all references to File $\mathrm{B}$ are classified as looping. Similar classification results are observed for references to File C.

PCC While the blocks of a sequential access detected by UBM has to be contiguous within a file, PCC considers sequential references as a set of distinct blocks that may belong to different files. The initial three blocks accessed by PC1 are classified as other and then PC1 is classified as sequential. Although PC2 is accessing the same set of blocks as PC1, it is still classified first as other and then as sequential when the threshold is reached. Before File $\mathrm{C}$ is accessed, the values of both seq and loop of PC1 are 6. Since seq of PC1 is increased and becomes larger than loop, accesses to File $\mathrm{C}$ made by $\mathrm{PC} 1$ are classified as sequential. Before File $\mathrm{C}$ is revisited by PC2, the values of both seq and loop of PC2 have changed to be 0 and 6 respectively through the references made by $\mathrm{PC} 1$, thus references to File $\mathrm{C}$ are detected as looping. After File $\mathrm{C}$ is accessed, the values of both seq and loop of PC2 are 6. References to File A made by PC2 are classified first as sequential and then as looping.

AMP The classification results are reported by the AMP simulator from its original author. To reduce the computation overhead, AMP uses a sampling method with some sacrifice to the detection accuracy. Since the sample trace used here is not large, the entire results are collected without using the sampling function in the AMP simulator. The initial recency of a PC, defined as the average ratios between the LRU stack positions and the stack length for all blocks accessed by the current $\mathrm{PC}$, is set to be 0.4. Last references to File A made by PC2 are incorrectly detected as other, which indicates that AMP has a tendency to classify looping references as other in the long term. We can use a shorter and simpler reference stream to further explain it. Given a looping reference stream $L=\{1,2,3,4,3,4,3,4\}$, the average recency of $L$ is 0.67 that is higher than the threshold, 0.4. Accordingly, AMP falsely considers the pattern of $L$ as other. In addition, AMP has another anomaly in which it has a tendency to erroneously classify a sequential stream as a looping one. For example, for a sequential reference stream $S=\{1,2,1,2,3,4,5,6,7,8\}$, the average recency of $S$ is 0 and AMP identifies this sequential pattern as looping. The first anomaly is more commonly observed in the workloads studies in this paper, which explains why the performance of AMP tends to be close to that of ARC in our experiments shown in Section V.

\section{Application Traces Used in the Simulation Study}

The traces used in this paper are obtained by using a trace collection tool provided by [7]. This tool is built upon the Linux strace utility that intercepts and records all system calls and signals of traced applications. A PC signature is obtained by tracing backwards the function call stack in strace. The modified strace investigates all I/O-related activities and reports 
TABLE I

TRACES USED AND THEIR STATISTICS

\begin{tabular}{|c||c|c|c|c|}
\hline Trace & Request Num. & Data Size (MB) & File Num. & PC Num. \\
\hline \hline gcc & 8765174 & 89.4 & 19875 & 69 \\
\hline gnuplot & 677442 & 121.8 & 8 & 26 \\
\hline cscope & 2131194 & 240 & 16613 & 40 \\
\hline glimpse & 2810992 & 194 & 16526 & 7 \\
\hline BLAST & 11042696 & 1789.6 & 13 & 20 \\
\hline tpch & 13468995 & 1187 & 49 & 150850 \\
\hline tpcr & 9415527 & 1087 & 49 & 150200 \\
\hline
\end{tabular}

\begin{tabular}{|c||c|c|c|c|c|}
\hline Trace & Concurrently Executed Applications & Request Num. & Data Size (MB) & File Num. & PC Num. \\
\hline \hline multi1 & glimpse + cscope & 4942186 & 434 & 33139 & 47 \\
\hline multi 2 & gnuplot + BLAST & 11720138 & 1911 & 21 & 46 \\
\hline multi3 & cscope + BLAST + gcc & 21939064 & 2119 & 36501 & 129 \\
\hline
\end{tabular}

the I/O triggering PC, file identifier(inode), I/O staring address and request size in bytes.

We use trace-driven simulations with various types of workloads to evaluate the RACE algorithm and compare it with other algorithms. These traces are considered typical and representative of applications in that most of them are routinely used in other caching algorithm studies. For example, the cscope, glimpse and gcc traces are used in [5], [7], [8], [17], [34], the gnuplot in [6], and tpch and tpcr in [35]. Table I summarizes the characteristics of these traces and more detailed description of each trace is presented below. The file and PC number represent the total number of unique files and pc signatures respectively.

1) gec is a GNU C compiler trace and it compiles and builds Linux kernel 2.6.10.

2) cscope [36] is an interactive utility that allows users to view and edit parts of the source code relevant to specified program items under the auxiliary of an index database. In cscope, an index database needs to be built first by scanning all examined source code. In our experiments, only the I/O operations during the searching phases are collected. The total size of the source code is $240 \mathrm{MB}$ and the index database is around 16MB.

3) glimpse [37] is a text information retrieval tool, searching for key words through large collections of text documents. It builds approximate indices for words and searches relatively fast with small index files. Similar to cscope, the I/O activities during the phase of index generation are not included in our collected trace. The total size of text is around $194 \mathrm{MB}$ and the glimpse index file is about 10MB.

4) gnuplot is a command-line driven interactive plotting program. Five figures are plotted by using four different plot functions that read data from two raw data files with sizes of $52 \mathrm{MB}$ and $70 \mathrm{MB}$, respectively.

5) BLAST [38] is a widely used scientific application in computational biology. It is designed to find regions of local similarity between a query sequence and all sequences in a large gene database. In this study, a large database named human EST is used and it is roughly $1.8 \mathrm{~GB}$ in size. Previous research has shown that the length of $90 \%$ of the query sequences used by biologists is within the range of 300600 characters [39]. Thus, in this work, we choose to use a sequence of 568 characters extracted from the ecoli.nt database as the query sequence.

6) tpch and tpcr benchmarks [40] perform random access to a few large MySQL database files. The traces used in this study are obtained from Ref. [35]. Ref. [35] suggests that disk I/O prefetching should be disabled in both tpch and tpcr to prevent cache pollution. Thus we only use the traces without prefetching.

7) multi1 is obtained by executing glimpse and cscope concurrently, which represents a text searching environment.

8) multi2 is obtained by executing gnuplot and BLAST concurrently, which simulates an environment of database queries and scientific visualization.

9) multi3 is obtained by concurrently executing three workloads, cscope, BLAST, and $g c c$, which provides a workload of database queries and code programming.

The traces of gnuplot, BLAST, gcc cscope, glimpse, tpch, and tpcr in Figure 2, Figure 3, and Figure 8, respectively, showing trace address as a function of the virtual time that is defined as the number of references issued so far and is incremented for each request. For the sake of visibility, the tpch and tpcr traces are shown with a sampling period of 400 .

\section{Performance Evaluation}

This section presents the performance evaluation of RACE through a trace-driven simulation study with ten different but typical traces from real applications. We compare the performance of RACE with seven other replacement algorithms, including UBM [5], [9], PCC [7], AMP [8], LIRS [17], [20], ARC [18], LRU and the off-line optimal policy (OPT). Simulation results of UBM, LIRS and AMP were obtained using simulators from their original authors respectively. We implemented the ARC algorithm according to the detailed pseudocode provided in [22]. We also implemented the PCC simulator and our RACE simulator by modifying the UBM simulator code. The UBM's cache management scheme based on the notion of marginal gain is used in PCC and RACE without any modification, which allows an effective and fair comparison of the pattern detection accuracies of UBM, PCC, and RACE.

The measure of hit ratio is used as our primary metric in the performance comparison. Hit ratio is defined as the fraction of $\mathrm{I} / \mathrm{O}$ requests that are successfully served by the cache without going off to the secondary disk storage. We believe that hit ratio is a 


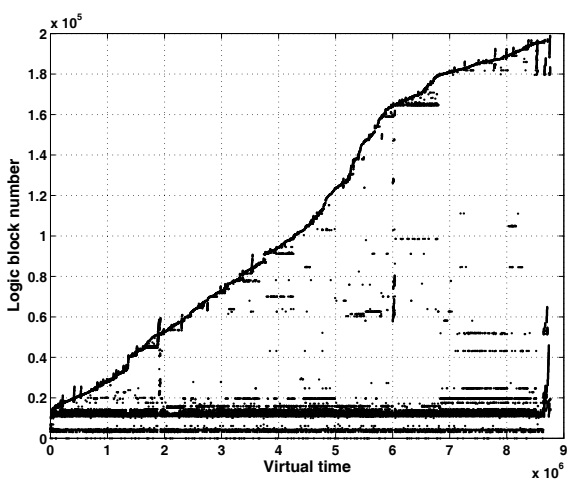

(a) gcc

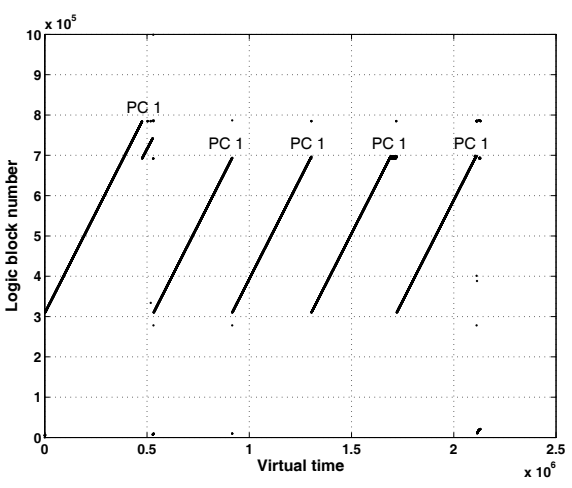

(b) cscope

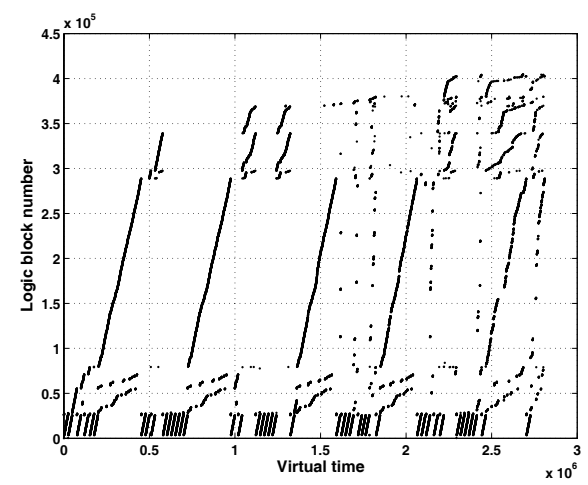

(c) glimpse

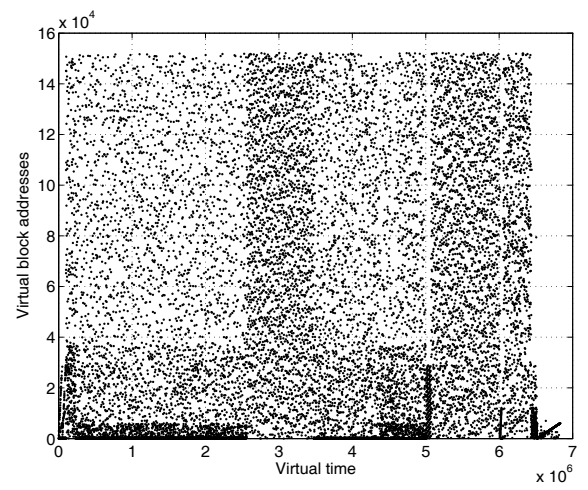

(d) tpch

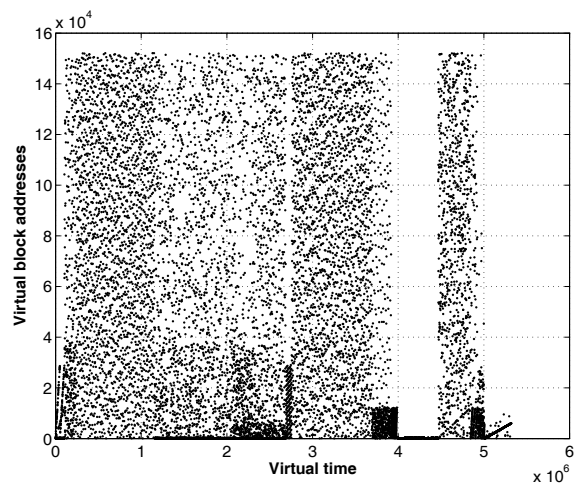

(e) tpcr

Fig. 8. Block references of cscope, gcc, glimpse, tpch, and tpcr

comprehensive metric to evaluate the accuracy, responsiveness, and stability of pattern-detection-based algorithms since these three factors can directly impact the hit ratios. More specifically, the accuracy of locality detection, in terms of access periods in this paper, directly influences the order of block eviction; Promptly adapting to patterns changes can avoid hit ratio degradation caused by obsolete information; Stability will guarantee consistently high hit ratios across different cache sizes and a wide spectrum of workloads.

\section{A. Cache Management Scheme}

PCC and RACE use the marginal gain in the original UBM [5], [9] simulator to manage the three partitions of the buffer cache. The marginal gain is defined as the expected extra hit ratios increased by adding one additional buffer [25], [41]. The marginal gain of the sequential partition is zero since no benefit can be obtained from caching one-time-use-only data. The marginal gain for the looping partition is $\frac{1}{p_{\max }+1}$ where $p_{\max }$ is the maximum looping period of blocks in the looping partition. The marginal gain for the other partition is estimated according to Belay's life function [42]. UBM, PCC and RACE aim to maximize the expected hit ratios by dynamically allocating the cache space to the three partitions: looping, sequential and other. For the sequential partition, no more than one buffer is allocated, except when the buffers are not fully utilized, since its marginal gain is zero. The cache space is switched between the looping and the other partitions according to the comparison of their estimated marginal gains. It always frees a buffer in the partition with a smaller marginal gain and allocates it to the other partition until both marginal gains converge to the same value.

AMP proposes a randomized eviction policy to manage the cache partitions. Upon a cache miss, AMP randomly chooses a non-empty partition and frees the block at the MRU position of that partition. While this randomized eviction works well for their design that employs ARC [18] to manage cache replacement, this replacement algorithm does not work efficiently for UBM, PCC and RACE for the simple reason that UBM, PCC, and RACE only use LRU or MRU to manage cache in order to achieve a low overhead. While ARC itself can automatically adapt to the workload changes, LRU and MRU do not provide such adaptability.

\section{B. Simulation Results}

Based on access patterns, the ten traces used in the simulation study are divided into two main groups. Traces gnuplot, BLAST, cscope fit in the group in which looping patterns dominate. Traces gcc, glimpse, tpch, tpcr, multil, multi2 and multi3 are in the group with mixed patterns. In what follows we report our simulation results in both groups and compare RACE with UBM, PCC, AMP, LIRS, ARC, LRU and OPT. The simulation parameters for these algorithms are given in Table II and all of them are suggested by their original authors in the literature except that the exponential average parameter $\alpha$ is not given in PCC [7].

\section{1) Performance under Workloads with Looping Patterns:}

- gnuplot Figure 9(a) shows the hit ratio comparisons for the workload gnuplot that has a looping pattern with long intervals. This workload generates a pathological case for 


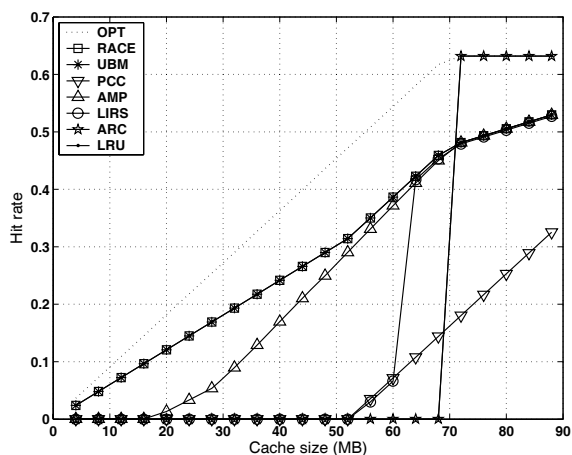

(a) gnuplot

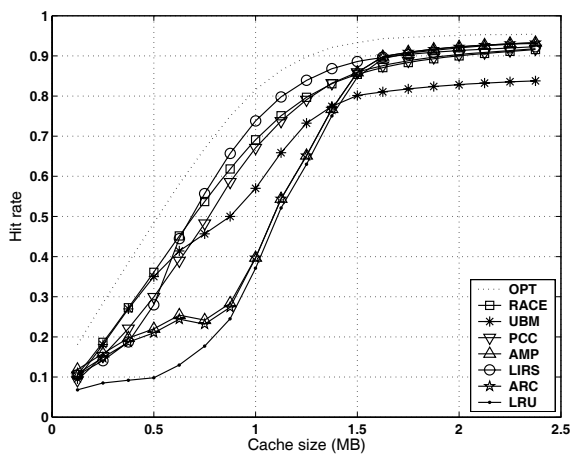

(d) $\mathrm{gcc}$

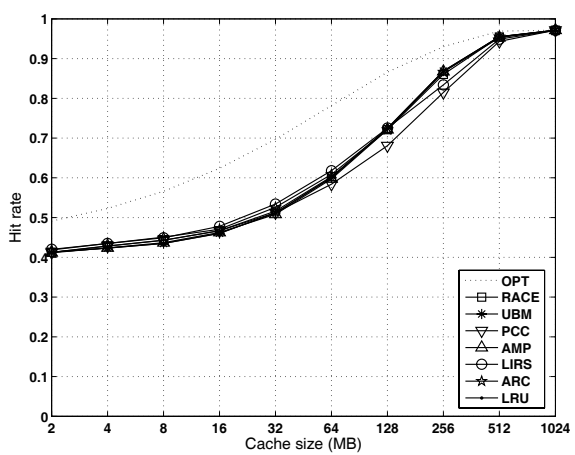

(g) tper

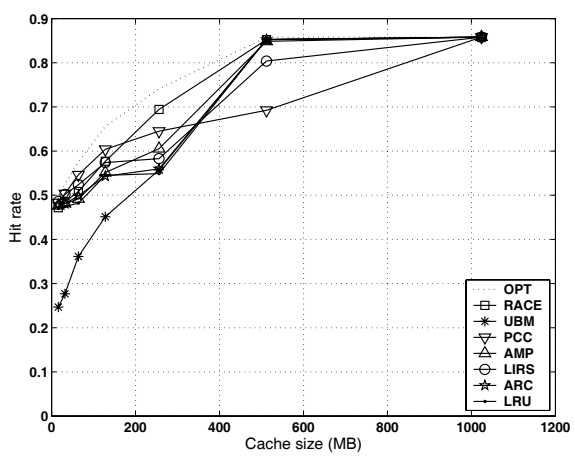

(j) multi3

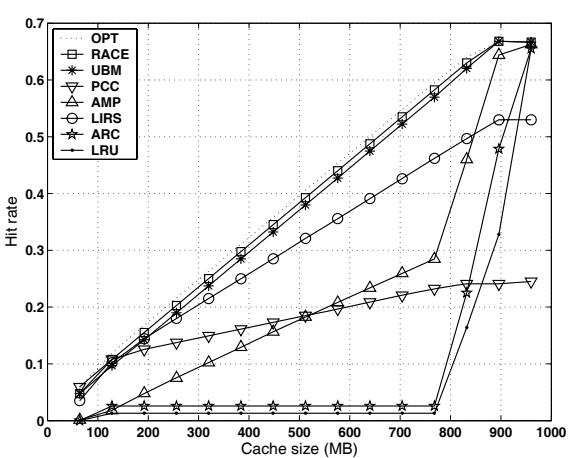

(b) BLAST

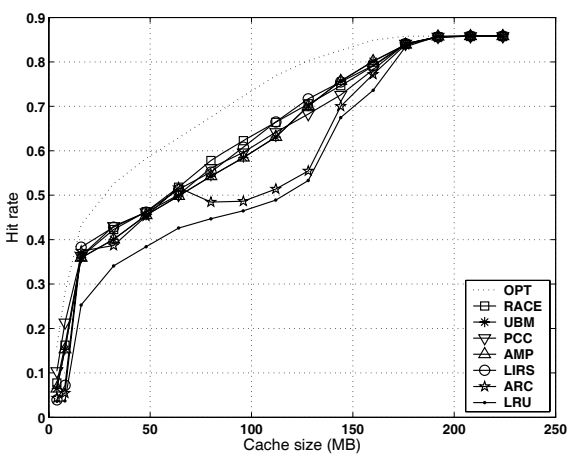

(e) glimpse

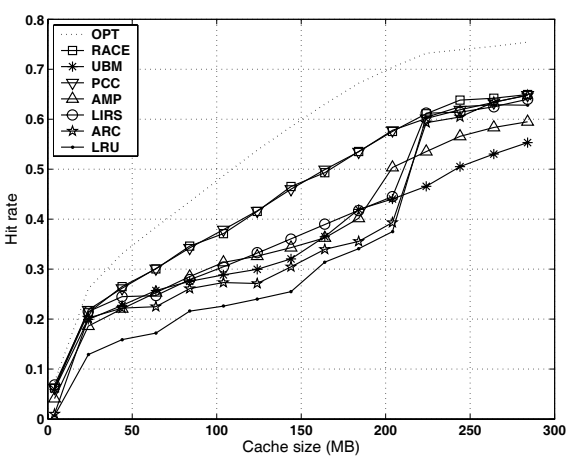

(h) multi1

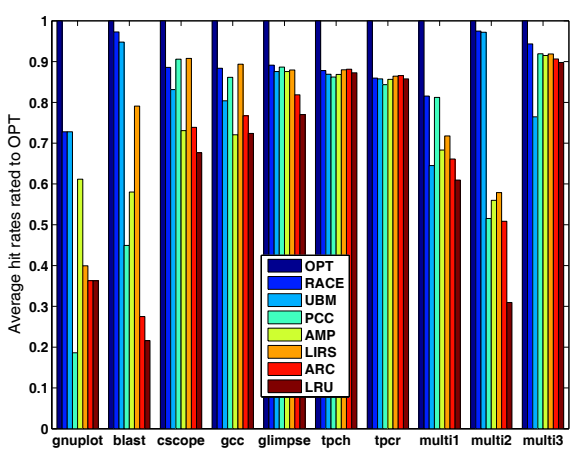
hit ratio of OPT)

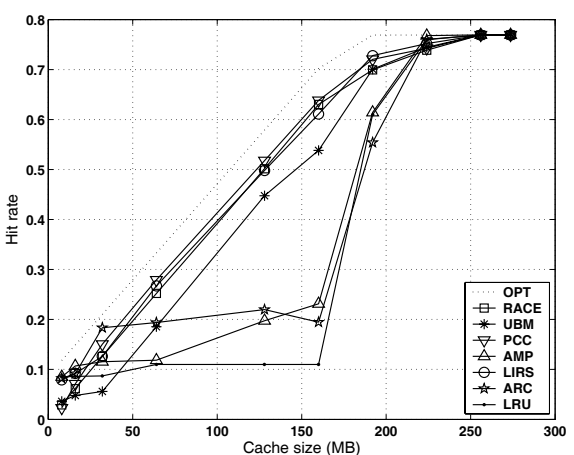

(c) cscope

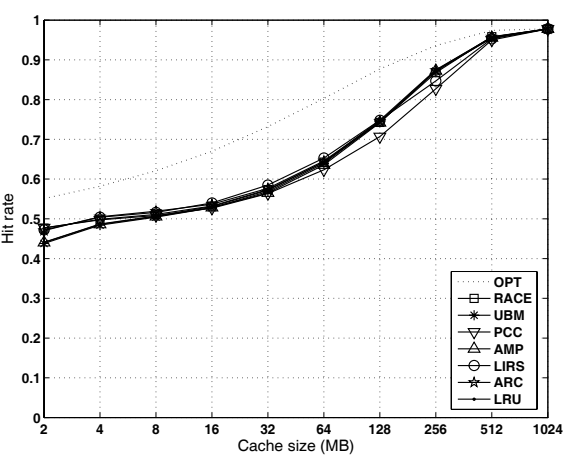

(f) tpch

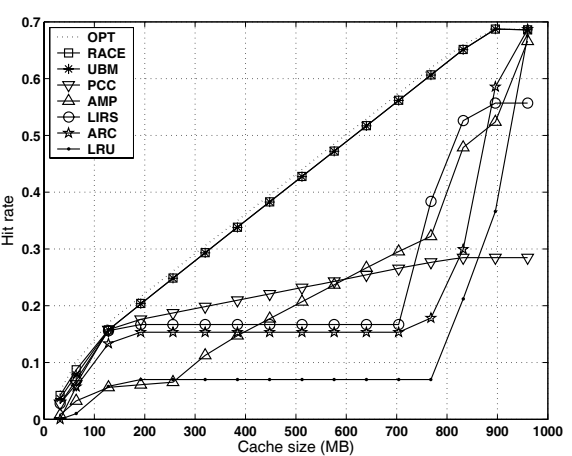

(i) multi2

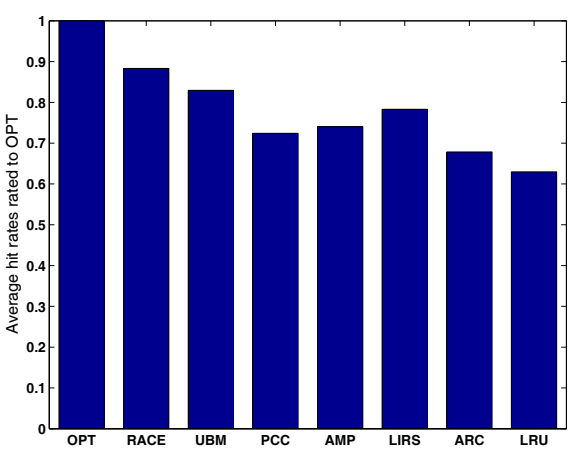

(1) Average hit ratios for all traces (normalized to the average hit ratio of OPT)

Fig. 9. Comparison of hit ratios 
TABLE II

PARAMETERS FOR CACHE REPLACEMENT POLICIES

\begin{tabular}{|c|c|}
\hline Policy & Parameters \\
\hline 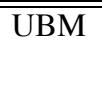 & $\begin{array}{l}\text { max loop or sequential lists }=2000, \text { threshold for detecting sequential access }=10, \\
\text { exponential average } \alpha=0.5\end{array}$ \\
\hline $\mathrm{PCC}$ & $\begin{array}{l}\text { exponential average } \alpha=0.5, \text { no sampling, threshold for detecting sequential access }= \\
100\end{array}$ \\
\hline AMP & $\begin{array}{l}\text { threshold for detecting looping access }=0.4 \text {, exponential average } \alpha=0.1 \text {, hit ratio } \\
\text { threshold for detecting sequential }=0.001\end{array}$ \\
\hline LIRS & $H I R=1 \%$ of cache, LRU stact $=2 \times$ cache size \\
\hline ARC & ghost cache $=$ cache size \\
\hline RACE & $\begin{array}{l}\text { size of the file hash table }=2000, \text { size of the PC hash table }=200, \alpha=0.5, \beta=0.1 \\
\text { and threshold for detecting sequential access }=100\end{array}$ \\
\hline
\end{tabular}

LRU when the size of accessed blocks in the loop is larger than the cache. Accordingly, LRU performs poorly and has the lowest hit ratios. Similar behavior is present in ARC as all blocks are accessed more than once and the frequency list is consequently managed by LRU. ARC achieves almost optimal hit rates when the cache is large since it can successfully evict out the least frequently used blocks. The benefit of LRU and ARC caching is only observed when the entire looping file set fits in the cache. Since the long sequential accesses are made by four PCs respectively as presented in Figure 2, PCC incorrectly classifies all references as sequential as expected and results in very low hit ratios. Both UBM and RACE, on the other hand, can correctly classify the references as looping after the first long sequence of sequential accesses, achieving much higher hit ratios. However, the cache management scheme employed in AMP is not efficient and thus resulting in lower performance than UBM and RACE. In sum, RACE achieves the same performance as UBM and a maximum of $52.3 \%$, and $39.9 \%$ improvement in hit ratio over LRU and AMP respectively.

- BLAST The performance comparisons under the BLAST workload is presented in Figure 9(b). The accesses are dominated by three major PCs initiated by three concurrently running threads in the BLAST application. PCC cannot detect the access sharing among these three program counters and marks many blocks as sequential. This is the main reason why the hit ratios of PCC are $20.8 \%, 19.8 \%$ and $5.0 \%$ lower than those of RACE, UBM and AMP respectively on average. Since there are only 13 files accessed in this trace, the misclassification of the first iteration of accesses to new files does not significantly degrade the UBM performance under this workload. Thus UBM achieves comparable hit ratios with RACE. While RACE improves LRU by as much as $56.9 \%$, for an average of $30.1 \%$, ARC only improves LRU by $15.1 \%$ at the maximum, with an average of $2.3 \%$. ARC is inherently capable of recording a reference history that is only twice the cache size. Under a workload with a large working set, such as BLAST, ARC fails to detect the looping patterns due to the lack of history information. Similarly, LIRS also suffers from limited history information that is stored in its two LRU stacks.

- cscope Figure 9(c) shows the hit ratio comparison for the cscope application. As explained in Section III, AMP tends to classify the looping references as other due to the fact that the average recency in AMP is highly sensitive to the stale history information as the center of working set shifts. As a result, the performance of AMP is close to that of ARC, which is used in AMP to manage the cache partition for the other pattern. PCC, LIRS and RACE achieves almost the same hit ratios and their hit ratios are $10.0 \%$ higher than that of UBM. Among the pattern-detection based algorithms, two main factors contribute to the inferior performance of UBM to RACE and PCC. First, with a total of 16613 files accessed in cscope, there are around $13.6 \%$ of files whose sizes are smaller than the threshold used in UBM. These small files form implicit looping patterns in a non-consecutive manner and thus are ignored in UBM. On the contrary, RACE and PCC can detect the implicit looping patterns in which a group of small files are repeatedly accessed. Secondly, there is around $5 \%$ of references that are issued to access files for the first time and UBM cannot make correct prediction for these references since UBM is intrinsically incapable of making accurate prediction when a file has not been accessed previously.

2) Performance under Workloads with Mixed Patterns:

- gcc Figure 9(d) shows the hit ratios under the workload of gcc that builds the newest version of the Linux kernel at the time of our experiments. Ref. [7] uses the trace collected only during pre-processing of an old Linux kernel (2.4.20). The trace does not reflect the whole I/O characteristics of building the kernel and it has only around 80000 read operations. We choose to use the trace that is collected during the whole building process of the newest Linux kernel 2.6.10 and it contains more than $9 \times 10^{6}$ read operations. We believe that this gives us a more comprehensive evaluation. In this trace, around $68 \%$ of the references are targeted at small files that are shorter than the threshold. When the cache size is smaller than $0.7 \mathrm{MB}$, UBM and RACE perform the best. However, the hit rates of LIRS are the highest when the cache size is between $0.7 \mathrm{MB}$ and $1.5 \mathrm{MB}$, although those of RACE come extremely close. Since the working set of $g c c$ is not large, LIRS can better differentiate the locality strength of referenced blocks and is $2.4 \%$ and $0.7 \%$ better than PCC and RACE respectively. AMP cannot adapt to the shift of working set centers and falsely classifies almost all references as others, which explains why AMP and ARC share similarly poor performance.

- glimpse Figure 9(e) shows the hits ratio comparisons under the glimpse workload. The performance of RACE, UBM, AMP, PCC and LIRS are very close to one another and 
perform much better than LRU. Specifically, RACE improves hit ratios of LRU by as much as $17.5 \%$, with an average of $8.1 \%$. Surprisingly, ARC clearly shows the Belady behavior where the hit ratio decreases while the cache size increases. This anomaly can be observed in the previous workloads as well. As introduced in Section II, ARC divides the cache of size $c$ into two LRU lists $L_{1}$ and $L_{2}$, and they retain a total of $c$ physical blocks and $c$ identifiers of recently evicted blocks. While blocks in $L_{1}$ have been used only once, blocks in $L_{2}$ have been used twice or more. A hit in $L_{1}$ promotes the referenced block to $L_{2}$ so that it can stay in the cache for a longer time. ARC bases its replacement strategy on the following assumption: if the requested block identifier is in $L_{1}$ on a cache miss, then it is likely that the number of physical blocks in $L_{1}$ is too small; Similarly if the identifier of a missed block is in $L_{2}$, then the number of physical blocks in $L_{2}$ is conjectured to be too small. Thus ARC adaptively allocates more cache space to a list that has more misses. It achieves this goal by dynamically changing the number of physical blocks allocated to $L_{1}$ with a variable step size. Under the same I/O workload, the step size is continuously updated by its exponential average and is influenced by the size of cache. Thus the step size cannot truly distinguish the "cold" blocks from the "hot" ones and leads to the severe Belady anomaly.

- tpch, tpcr While the tpch and tpcr benchmarks repeatedly access a total of six large database files, only $3 \%$ references occur to immediately consecutive blocks [35] and over $80 \%$ stride distances between consecutive references are larger than 10 blocks. Figures $9(\mathrm{f})$ and $9(\mathrm{~g})$ compare the hit ratio performance. The PCC performs slightly worse than LRU when the cache size is larger than $64 \mathrm{MB}$. While the majority of references are absorbed by the six databases, there are over $15 \mathrm{~K}$ program signatures and the mis-prediction of access periods degrades the performance in PCC. Compared with PCC, RACE not only correctly identifies more periodical accesses, but also provides more accurate access periods.

- multi1, multi2, multi3 The hit ratio comparisons under the workloads of multil, multi2 and multi3 are presented in Figures 9(h), 9(i) and 9(j) respectively. In sum, in multil, RACE and PCC achieve the best hit ratios. In multi2, the hit ratios of RACE and UBM are the highest. RACE outperforms all other algorithms in multi3.

\section{Average Hit Ratio Comparisons}

Figure $9(\mathrm{k})$ shows the average hit ratios normalized to the average hit ratio of the OPT replacement algorithm for the eight workloads studied in this paper. Figure 10 compares the classification results between RACE, UBM, PCC, and AMP, which helps explain the reasons behind the superiority of RACE in terms of hit ratios. For gnuplot, BLAST and multi2, the PCC algorithm is pathological in that PCC cannot distinguish the pattern sharing among different PCs and falsely classifies many looping patterns as sequential patterns, as shown in Figure 10. For cscope, gcc, glimpse, multil, and multi3, the UBM algorithm is pathological since it ignores the patterns clearly exhibited in small files and it is incapable of correctly detecting the access patterns for files that have not been referenced before. In almost all workloads, AMP erroneously identifies a larger fraction of accesses as the other pattern, as much as $74.9 \%, 19.7 \%$, and
$74.9 \%$ on average more than RACE, UBM, and PCC respectively, which dramatically lowers its hit ratios. RACE, on the contrary, exploits the detection mechanism in both the continuous block address space within files and the discrete block address space in program contexts, resulting in an average of $52.4 \%, 24.5 \%$, and $70.8 \%$ more looping patterns being correctly detected than UBM, PCC and AMP respectively.

The experimental results on the ten workloads show that RACE is more robust than all other algorithms. Figure 9(1) presents the average values of hit ratios (normalized to the optimal hit ratios) presented in Figure $9(\mathrm{k})$. Compared with UBM, PCC, AMP, LIRS, ARC, LRU, the normalized hit ratio of RACE is higher by an average of $5.4 \%, 15.9 \%, 14.3 \%, 10.0 \%, 20.5 \%$, and $25.4 \%$, respectively. Table III shows the arithmetic average of absolute hit ratios of these algorithms with different sizes in each workload. RACE can successfully overcome the drawbacks of LRU and improve its absolute hit ratios by as much as $56.9 \%$, with an average of $15.5 \%$.

Compared with other state-of-the-art pattern-detection based schemes, RACE outperforms UBM, PCC and AMP by as much as $22.5 \%, 42.7 \%$ and $39.9 \%$, with an average of $3.3 \%, 6.6 \%$ and $6.9 \%$, respectively. In the cscope trace, RACE is $1.0 \%$ inferior to PCC on average due to the following fact: Although RACE correctly classifies files accessed at the end of first iteration as looping, these files are only accessed twice, as shown in Figure 8(b), and RACE wastes partial memory by caching them. Compared with the state-of-the-art recency/frequency based schemes, RACE consistently beats ARC in all workloads and outperforms LIRS in most workloads except cscope and gcc. In the cscope and gcc traces, RACE is on average $1.1 \%$ and $0.7 \%$ inferior to LIRS in absolute hit ratio. Since RACE improves the hit ratios of LIRS with an average of $6.0 \%$ over the eight workloads, we conclude that such slight performance degradation in cscope and gcc is not severe. The $g c c$ workload is extremely LRU-friendly, in which 89.4 MB data is accessed and a LRU cache with a size of 1.5MB can achieve a hit ratio of $86 \%$. It is our future work to avoid such slight performance degradation by improving our detection algorithm or by incorporating LIRS into RACE to manage the cache partitions. In sum, RACE improves the hit ratios of UBM, PCC, AMP, LIRS, ARC, and LRU relatively by $6.8 \%, 14.6 \%$, $15.2 \%, 8.7 \%, 21.7 .3 \%$ and $29.3 \%$ on average. This superiority indicates that our RACE scheme is more robust and adaptive than any of the other six caching schemes and also proves our assumption that the future access patterns are highly correlated with both program contexts and requested data.

\section{Sensitivity Study on the Sampling Frequency}

Our RACE algorithm needs to update both the file hash table and the PC hash table. The cache sizes are $1 \mathrm{MB}$ for Linux, $500 \mathrm{MB}$ for mult1, mult2, and mult3, and 50MB for the others. Figure 11 shows the sensitivity of the updating frequency. With a sampling frequency of less than 16 blocks, the hit ratios are barely adversely affected for most benchmarks except for $g c c$ and cscope. In gnuplot, the performance of RACE reduces to LRU when the sampling frequency is large. From this analysis, we believe that the updating overhead and the memory requirement of the file hash tables and the PC tables can be traded off through appropriate sampling. A sampling period of 16 blocks provides a good tradeoff for the studied benchmarks. 


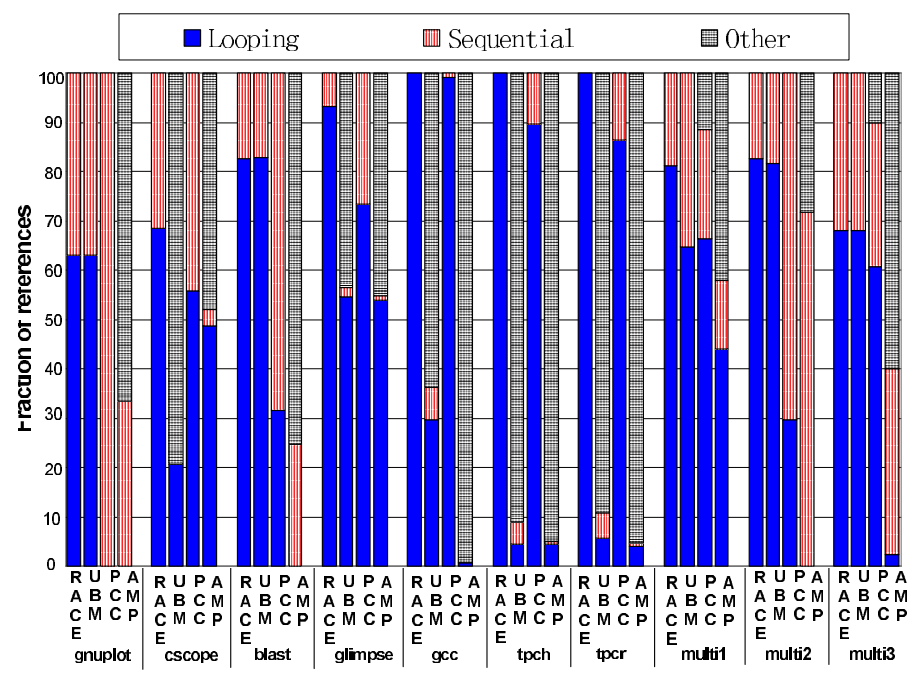

Fig. 10. Comparisons of classification results

TABLE III

Hit RATIOS UNDER THE 8 TRACES, AVERAGE OVER DIFFERENT CACHE SIZES

\begin{tabular}{|l||c|c|c|c|c|c|c|c|}
\hline & OPT & RACE & UBM & PCC & AMP & LIRS & ARC & LRU \\
\hline \hline gnuplot & 0.3961 & $\mathbf{0 . 2 8 8 4}$ & $\mathbf{0 . 2 8 8 4}$ & 0.0738 & 0.2423 & 0.1582 & 0.1437 & 0.1436 \\
\hline BLAST & 0.3982 & $\mathbf{0 . 3 8 7 2}$ & 0.3773 & 0.1789 & 0.2311 & 0.3149 & 0.1094 & 0.0860 \\
\hline cscope & 0.5167 & 0.4577 & 0.4295 & 0.4681 & 0.3776 & $\mathbf{0 . 4 6 9 1}$ & 0.3818 & 0.3496 \\
\hline gcc & 0.7587 & 0.6704 & 0.6100 & 0.6536 & 0.5468 & $\mathbf{0 . 6 7 8 1}$ & 0.5820 & 0.5492 \\
\hline glimpse & 0.6681 & $\mathbf{0 . 5 9 5 4}$ & 0.5849 & 0.5923 & 0.5849 & 0.5874 & 0.5469 & 0.5144 \\
\hline tpch & 0.7724 & 0.6781 & 0.6711 & 0.6659 & 0.6709 & 0.6796 & $\mathbf{0 . 6 8 0 7}$ & 0.6739 \\
\hline tpcr & 0.7419 & 0.6378 & 0.6363 & 0.6257 & 0.6354 & 0.6413 & $\mathbf{0 . 6 4 2 3}$ & 0.6364 \\
\hline multil & 0.5381 & $\mathbf{0 . 4 3 8 9}$ & 0.3471 & 0.4371 & 0.3676 & 0.3863 & 0.3558 & 0.3280 \\
\hline multi2 & 0.4081 & $\mathbf{0 . 3 9 7 8}$ & 0.3967 & 0.2104 & 0.2285 & 0.2363 & 0.2075 & 0.1263 \\
\hline multi3 & 0.6734 & $\mathbf{0 . 6 3 5 3}$ & 0.5148 & 0.6190 & 0.6161 & 0.6187 & 0.6103 & 0.6047 \\
\hline overall & 0.5872 & $\mathbf{0 . 5 1 8 7}$ & 0.4856 & 0.4525 & 0.4501 & 0.4770 & 0.4261 & 0.4012 \\
\hline
\end{tabular}

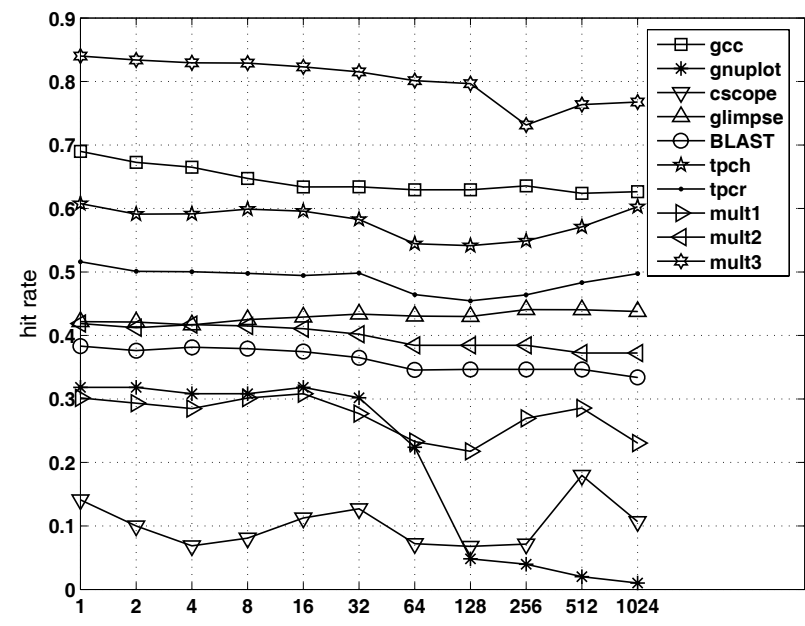

Fig. 11. Impacts of sampling frequency on hit ratios

\section{CONCLUSIONS}

Cache replacement algorithms are crucial in bridging the increasing performance gap between processors and disk drives. Motivated by the limitations of existing state-of-the-art cache replacement algorithms, we propose a novel and simple block replacement algorithm called RACE. We make three main contributions: 1) We collected the I/O traces for eight real applications and investigate I/O access patterns in two correlated spaces: the program context space from which I/O operations are issued, and the file space to which I/O requests are addressed. 2) Our comprehensive application trace study revealed the pathological behavior in existing state-of-the-art cache replacement algorithms, including a file-level detection method (UBM) and two program context level detection methods (PCC and AMP). 3) Extensive simulation study conducted under these real-application workloads demonstrated that RACE, through its exploitation of the detection mechanism in both the continuous block address space within files and the discrete block address space in program contexts, is able to accurately detect reference patterns from both the file level and the program context level and thus significantly outperforms other state-of-the-art recency/frequency based algorithms and pattern-detection based algorithms. Due to the very high buffer cache miss penalties, which are typically 6 orders of magnitude higher than buffer cache hit times, we believe that the significant gains in hit ratios obtained by RACE over other algorithms will likely have significant performance implications in application response times.

Our study has two limitations. First, we have not implemented our design and evaluated it in real systems. Compared with 
recency/frequency based algorithms such as LRU, LIRS, and $\mathrm{ARC}$, the program-context based algorithms, including RACE, PCC and AMP, need to pay the extra overhead of obtaining program counter signatures. Ref. [43] reports that it is inefficient to obtain program signatures through stack traversals in their quick-hack implementation. It is suggested that a library modification approach, which can read the PC directly from the calling program's stack and hence requires the least amount of overhead. Secondly, in order to achieve a direct comparison of pattern detection accuracy, RACE, as well as PCC, uses the marginal gain functions proposed in the UBM scheme to dynamically allocate the buffer cache. We believe that a more effective allocation scheme will be helpful to further improve the hit ratios. In the future, we will implement RACE into Linux systems and investigate other efficient allocation schemes.

\section{ACKNOWLEDGMENTS}

We are grateful to Dr. Song Jiang and Dr. Xiaodong Zhang for providing LIRS cache simulator, Feng Zhou et. al. for their AMP simulator and Chris Gniady et al. for their trace collection tool and tpch and tpcr traces. The authors would like to thank the anonymous reviewers for their efforts in improving this paper. This work is supported by a UMaine Startup Grant, NSF Grant CCF-0621493, NSF Grant CCF-0621526, and a Chinese NSF 973 Project Grant (No. 2004cb318201).

\section{REFERENCES}

[1] M. J. Bach, The design of the UNIX operating system. Upper Saddle River, NJ, USA: Prentice-Hall, Inc., 1986.

[2] A. S. Tanenbaum and A. S.Woodhull, Operating Systems Design and Implementation. Upper Saddle River, NJ, USA: Prentice-Hall, Inc., 1987.

[3] R. W. Carr and J. L. Hennessy, "WSCLOCK - a simple and effective algorithm for virtual memory management," in Proceedings of the eighth ACM symposium on Operating systems principles (SOSP). New York, NY, USA: ACM Press, 1981, pp. 87-95.

[4] A. J. Smith, "Analysis of the optimal, look-ahead demand paging algorithms," vol. 5, no. 4, pp. 743-757, Dec. 1976.

[5] J. M. Kim, J. Choi, J. Kim, S. H. Noh, S. L. Min, Y. Cho, and C. S. Kim, "A low-overhead, high-performance unified buffer management scheme that exploits sequential and looping references," in 4th Symposium on Operating System Design and Implementation (OSDI), Oct. 2000, pp. 119-134.

[6] J. Choi, S. H. Noh, S. L. Min, and Y. Cho, "An implementation study of a detection-based adaptive block replacement scheme," in Proceedings of the 1999 USENIX Annual Technical Conference, Jun. 1999, pp. 239252.

[7] C. Gniady, A. R. Butt, and Y. C. Hu, "Program-counter-based pattern classification in buffer caching." in Proceedings of 6th Symposium on Operating System Design and Implementation (OSDI), Dec. 2004, pp. 395-408.

[8] F. Zhou, R. von Behren, and E. Brewer, "AMP: Program context specific buffer caching," in Proceedings of the USENIX Technical Conference, Apr. 2005.

[9] J. Choi, S. H. Noh, S. L. Min, E.-Y. Ha, and Y. Cho, "Design, implementation, and performance evaluation of a detection-based adaptive block replacement scheme," IEEE Trans. Comput., vol. 51, no. 7, pp. 793-800, 2002.

[10] L. A. Belady, "A study of replacement algorithms for a virtual-storage computer," IBM Systems Journal, vol. 5, no. 2, pp. 78-101, 1966.

[11] R. L. Mattson, J. Gecsei, D. R. Slutz, and I. L. Traiger, "Evaluation techniques for storage hierarchies." IBM Systems Journal, vol. 9, no. 2, pp. 78-117, 1970.

[12] E. J. O'Neil, P. E. O'Neil, and G. Weikum, "The lru-k page replacement algorithm for database disk buffering," in Proceedings of the 1993 ACM SIGMOD international conference on Management of data. New York, NY, USA: ACM Press, 1993, pp. 297-306.
[13] T. Johnson and D. Shasha, "2Q: A low overhead high performance buffer management replacement algorithm," in Proceedings of the 20th International Conference on Very Large Data Bases (VLDB). San Francisco, CA, USA: Morgan Kaufmann Publishers Inc., 1994, pp. 439450.

[14] D. Lee, J. Choi, J.-H. Kim, S. H. Noh, S. L. Min, Y. Cho, and C. S. Kim, "On the existence of a spectrum of policies that subsumes the least recently used (LRU) and least frequently used (LFU) policies," in Proceedings of the 1999 ACM SIGMETRICS International conference on Measurement and Modeling of Computer Systems, 1999, pp. 134-143.

[15] Y. Smaragdakis, S. Kaplan, and P. Wilson, "EELRU: simple and effective adaptive page replacement," in Proceedings of the 1999 ACM SIGMETRICS international conference on Measurement and modeling of computer systems, New York, NY, USA, 1999, pp. 122-133.

[16] Y. Zhou, J. Philbin, and K. Li, "The multi-queue replacement algorithm for second level buffer caches," in Proceedings of the General Track: 2002 USENIX Annual Technical Conference. Berkeley, CA, USA: USENIX Association, 2001, pp. 91-104.

[17] S. Jiang and X. Zhang, "LIRS: An efficient low inter-reference recency set replacement policy to improve buffer cache performance," in Proceedings of the ACM SIGMETRICS Conference on Measurement and Modeling of Computer Systems, Jun. 2002, pp. 31-42.

[18] N. Megiddo and D. S. Modha, "ARC: A self-tuning, low overhead replacement cache," in Proceedings of the 2nd USENIX Conference on File and Storage Technologies (FAST), Mar. 2003, pp. 115-130.

[19] D. Lee, J. Choi, J.-H. Kim, S. H. Noh, S. L. Min, Y. Cho, and C. S. Kim, "LRFU: A spectrum of policies that subsumes the least recently used and least frequently used policies," IEEE Transactions on Computer, vol. 50, no. 12, pp. 1352-1361, 2001.

[20] J. Song and Z. Xiaodong, "Making LRU friendly to weak locality workloads: A novel replacement algorithm to improve buffer cache performance," IEEE Transactions on Computers, vol. 54, no. 8, pp. 939952,2005

[21] S. Jiang, F. Chen, and X. Zhang, "CLOCK-Pro: an effective improvement of the CLOCK replacement," in Proceedings of 2005 USENIX Annual Technical Conference, Apr. 2005.

[22] N. Megiddo and D. S. Modha, "One up on LRU," ;login: - The Magazine of the USENIX Association, vol. 4, no. 18, pp. 7-11, 2003.

[23] S. Bansal and D. S. Modha, "CAR: Clock with adaptive replacement," pp. 187-200, Mar. 2004.

[24] P. Cao, E. W. Felten, A. R. Karlin, and K. Li, "Implementation and performance of integrated application-controlled file caching, prefetching, and disk scheduling," ACM Transactions on Computer Systems, vol. 14, no. 4, pp. 311-343, 1996.

[25] R. H. Patterson, G. A. Gibson, E. Ginting, D. Stodolsky, and J. Zelenka, "Informed prefetching and caching," in Proceedings of the fifteenth ACM symposium on Operating systems principles (SOSP). New York, NY, USA: ACM Press, 1995, pp. 79-95.

[26] A. D. Brown, T. C. Mowry, and O. Krieger, "Compiler-based I/O prefetching for out-of-core applications," ACM Transactions on Computer Systems, vol. 19, no. 2, pp. 111-170, 2001.

[27] T. M. Madhyastha and D. A. Reed, "Learning to classify parallel input/output access patterns," IEEE Trans. Parallel Distrib. Syst., vol. 13, no. 8 , pp. $802-813,2002$

[28] G. Glass and P. Cao, "Adaptive page replacement based on memory reference behavior," in Proceedings of the ACM SIGMETRICS international conference on Measurement and Modeling of Computer Systems. New York, NY, USA: ACM Press, 1997, pp. 115-126.

[29] K. So and R. N. Rechtschaffen, "Cache operations by MRU change." IEEE Trans. Computers, vol. 37, no. 6, pp. 700-709, 1988.

[30] R. Floyd, "Short-term file reference patterns in a UNIX environment," Computer Science Department, University of Rochester, Rochester, NY, Tech. Rep. TR-177, Mar. 1986.

[31] C. Staelin, "High performance file system design," Ph.D. dissertation, Department of Computer Science, Princeton University, Oct. 1991.

[32] V. Cate and T. Gross, "Combining the concepts of compression and caching for a two-level file system," in Proceedings Architectural Support for Programming Languages and Operating Systems (ASPLOS), Santa Clara, CA, Apr. 1991, pp. 200-211.

[33] H. Tang and T. Yang, "An efficient data location protocol for selforganizing storage clusters," in Proceedings of ACM/IEEE SuperComputing (SC), Phoenix, AZ, USA, Nov. 2003.

[34] P. Cao, E. W. Felten, and K. Li, "Application-controlled file caching policies," in USENIX Summer Technical Conference, Jun. 1994, pp. 171182.

[35] A. R. Butt, C. Gniady, and Y. C. Hu, "The performance impact of kernel prefetching on buffer cache replacement algorithms," in Proceedings 
of the International Conference on Measurements and Modeling of Computer Systems(SIGMETRICS). ACM, Jun. 2005, pp. 157-168.

[36] J. L. Steffen, "Interactive examination of a C program with Cscope," in Proceedings of Winter USENIX Technical Conference, Jan. 1985.

[37] U. Manber and S. Wu, "GLIMPSE: A tool to search through entire file systems," in Proceedings of Winter USENIX Technical Conference, San Francisco, CA, USA, 1994, pp. 23-32.

[38] S. F. Altschul, W. Gish, W. Miller, E. W. Myers, and D. J. Lipman, "Basic local alignment search tool." J Mol Biol, vol. 215, no. 3, pp. 403-410, October 1990. [Online]. Available: http://dx.doi.org/10.1006/jmbi.1990.9999

[39] K. T. Pedretti, T. L. Casavant, R. C. Braun, T. E. Scheetz, C. L. Birkett, and C. A. Roberts, "Three complementary approaches to parallelization of local blast service on workstation clusters (invited paper)," in Proceedings of the 5th International Conference on Parallel Computing Technologies (PACT). London, UK: Springer-Verlag, 1999, pp. 271282.

[40] TPC, "Transaction Processing Council," Website, http://www.tpc.org.

[41] D. Thiebaut, H. S. Stone, and J. L. Wolf, "Improving disk cache hit-ratios through cache partitioning," IEEE Transaction on Computer, vol. 41, no. 6, pp. 665-676, 1992.

[42] J. R. Spirn, Program Behavior: Models and Measurements. New York, NY, USA: Elsevier Science Inc., 1977.

[43] C. Gniady, A. R. Butt, Y. C. Hu, and Y.-H. Lu, "Program counter-based prediction techniques for dynamic power management," IEEE Trans. Comput., vol. 55, no. 6, pp. 641-658, 2006.

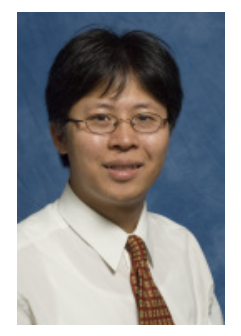

Yifeng Zhu Yifeng Zhu received his B.Sc. degree in Electrical Engineering in 1998 from Huazhong University of Science and Technology, Wuhan, China; the M.S. and Ph.D. degree in Computer Science from University of Nebraska - Lincoln in 2002 and 2005 respectively. He is an assistant professor in the Electrical and Computer Engineering department at University of Maine. His main research interests are parallel I/O storage systems, cluster computing, grid computing, and computer architecture and systems. Computer Society, and the Francis Crowe Society.

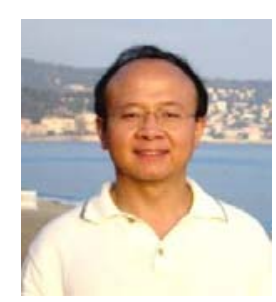

Hong Jiang Hong Jiang received the B.Sc. degree in Computer Engineering in 1982 from Huazhong University of Science and Technology, Wuhan, China; the M.A.Sc. degree in Computer Engineering in 1987 from the University of Toronto, Toronto, Canada; and the $\mathrm{PhD}$ degree in Computer Science in 1991 from the Texas A\&M University, College Station, Texas, USA. Since August 1991 he has been at the University of Nebraska-Lincoln, Lincoln, Nebraska, USA, where he is Professor and Vice Chair in the Department of Computer Science and Engineering. His present research interests are computer architecture, computer storage systems and parallel I/O, parallel/distributed computing, cluster and Grid computing, performance evaluation, real-time systems, middleware, and distributed systems for distance education. He has over 130 publications in major journals and international Conferences in these areas and his research has been supported by NSF, DOD and the State of Nebraska. Dr. Jiang is a Member of ACM, the IEEE Computer Society, and the ACM SIGARCH. 